\title{
Utilization of Primary and Secondary Alcohols by the Detergent-degrading Bacterium Pseudomonas C12B
}

\author{
By DAVID J. RIGBY, KENNETH S. DODGSON AND GRAHAM F. WHITE* \\ Department of Biochemistry, University College, $P O$ Box 78 , \\ Cardiff CF1 IXL, UK
}

(Received 25 June 1985; revised 9 August 1985)

\begin{abstract}
The capacity of the detergent-degrading bacterium Pseudomonas $\mathrm{C} 12 \mathrm{~B}$ to grow on, and oxidize, a range of primary and secondary alcohols of chain lengths from $C_{2}$ to $C_{16}$ was examined. The organism was able to grow on most primary alcohols tested, and especially well on the $\mathrm{C}_{10}-\mathrm{C}_{12}$ homologues. Of several secondary alcohols, $\mathrm{C}_{11}-\mathrm{C}_{13}$ homologues of alkan-2-ols supported good growth, as did some long-chain symmetrical or near symmetrical alcohols. There was constitutive synthesis of primary and alkan-2-ol dehydrogenase activities, enabling the organism to oxidize primary alcohols and alkan-2-ols up to at least $C_{11}$. Experiments with resolved $D$ - and L-octan-2-ols suggested that the constitutive alkan-2-ol dehydrogenase activity was specific for D-isomers. Growth on secondary alcohols induced synthesis of L-alkan-2-ol dehydrogenase(s) and also greatly increased the capacity of extracts to oxidize racemic alkan-2ols in the range $C_{6}-C_{11}$. An enzyme with high activity towards symmetrical alcohols was also induced by growth on secondary (especially symmetrical) alcohols. Collectively, the various alcohol dehydrogenase activities detected would enable Pseudomonas $\mathrm{C} 12 \mathrm{~B}$ to oxidize all the alcohols liberated from mixed alkyl sulphate detergents by the organism's complement of two primary and three secondary alkylsulphatases.
\end{abstract}

\section{INTRODUCTION}

Pseudomonas $\mathrm{C12B}$, a detergent-degrading bacterium originally isolated from soil near a sewage outfall (Payne \& Feisal, 1963), is able to degrade primary and secondary alkyl sulphate, alkanesulphonate and alkyltriethoxy sulphate surfactants. Degradation of the alkyl sulphates is initiated by the action of five alkylsulphatase enzymes (Dodgson \& White, 1983). Two of these enzymes (designated $\mathrm{P} 1$ and $\mathrm{P} 2$ ) are active towards primary alkyl sulphates of chain length $\mathrm{C}_{6}$ or greater (e.g. sodium dodecyl sulphate, SDS) whereas the remaining three enzymes (S1, S2 and $\mathrm{S} 3$ ) are secondary alkylsulphatases. The $\mathrm{S} 1$ and $\mathrm{S} 2$ enzymes are active towards $\mathrm{D}$ - and L-isomers, respectively, of alkan-2-sulphates of chain length $\mathrm{C}_{6}$ or greater. The $\mathrm{S} 3$ enzyme is active towards symmetrical (e.g. nonan-5-sulphate) or near-symmetrical alkyl sulphates.

Primary alcohols liberated by the action of the P1 and P2 enzymes are considered (Dodgson \& White, 1983; Williams et al., 1966) to undergo oxidation via aldehydes to carboxylic acids which in turn serve as substrates for $\beta$-oxidation. Metabolism of secondary alcohols is more complicated, requiring $\mathrm{C}-\mathrm{C}$ bond cleavage to produce substrates for $\beta$-oxidation (Lijmbach \& Brinkhuis, 1973), but oxidation of the secondary hydroxyl groups to ketones is still the first step. Pseudomonas $\mathrm{C} 12 \mathrm{~B}$ can grow on, and oxidize, linear primary alcohols with even- and oddnumbered carbon-chains from $C_{2}$ to $C_{11}$ (Payne, 1963; Williams et al., 1966). Moreover, cell extracts contained NAD-linked dehydrogenase(s) active towards these primary, and certain secondary, alcohols.

Studies on Comamonas terrigena, a co-isolate of Pseudomonas C12B and taxonomically related to Pseudomonas testosteroni and Pseudomonas acidovorans (Hugh, 1965; Stanier et al., 1966; Palleroni et al., 1973), have shown that this organism produces two alkylsulphatases only, one of 
which (designated CS1) acts on L-alkan-2-sulphates of chain length $\mathrm{C}_{6}$ or greater, and the other (CS2) on the corresponding D-isomers (Dodgson \& White, 1983; Matcham et al., 1977; Barrett et al., 1980a). Like the S1, S2 and S3 secondary alkylsulphatases, the two $C$. terrigena enzymes operate by $\mathrm{C}-\mathrm{O}$ bond fission with inversion of configuration at the esterified carbon atom, so that $\mathrm{D}$-esters are converted to L-alkan-2-ols and vice versa. The sulphatase stereospecificity is matched by a pair of alcohol dehydrogenases, one of which acts on D-alkan-2-ols and primary alcohols, and the other on L-alkan-2-ols, symmetrical secondary alcohols and probably other positional isomers of secondary alcohols of the L-configuration (Barrett et al., 1980 b; Barrett et al., 1981).

In view of the complementarity between alcohol dehydrogenases and alkylsulphatases in $C$. terrigena, the question now arises as to whether a similar situation obtains in Pseudomonas C12B. Because the latter organism has a more versatile complement of alkylsulphatases, as well as a much greater capacity for detergent degradation, it might be expected that a greater variety of alcohol dehydrogenases might also be present. The present work was undertaken to investigate the ability of Pseudomonas C12B to grow on a variety of primary and secondary alcohols and to attempt to relate this ability as far as possible to the alcohol dehydrogenase activities produced by the organism.

\section{METHODS}

Chemicals. Nutrient broth and agar were obtained from Difco. Primary and secondary alcohols for growth experiments and enzyme assays were obtained from Aldrich, BDH, Eastman, Fluka, ICN, K and K and Koch Light. SDS purchased from $\mathrm{BDH}$ was recrystallized from methanol before use. All other reagents were obtained from BDH or Sigma.

Organism and growth. The isolation of Pseudomonas C12B (NCIB 11753) has been described elsewhere (Payne \& Feisal, 1963). The organism has been maintained (in this laboratory since 1972) by monthly transfers on nutrient agar containing $0.8 \%(\mathrm{w} / \mathrm{v})$ nutrient broth solidified with $1.5 \%(\mathrm{w} / \mathrm{v})$ agar. Basal salts medium was prepared as described by Fitzgerald \& Payne (1972) with the addition of $0.014 \% \mathrm{Na}_{2} \mathrm{SO}_{4}$. Nutrient broth $(0 \cdot 8 \%)$ and basal salts alone or containing $1 \%(\mathrm{w} / \mathrm{v})$ pyruvate or undecanoate were autoclaved whole, but all alcohols were added aseptically $(0.1 \%)$ to autoclaved media. For growth experiments, $250 \mathrm{ml}$ Erlenmeyer flasks containing $50 \mathrm{ml}$ of medium were inoculated $(0.02 \%)$ with a 24 h nutrient broth culture and shaken (100 r.p.m.) at $30{ }^{\circ} \mathrm{C}$. After 24 h, culture optical densities were measured at $650 \mathrm{~nm}$ (broth) or $420 \mathrm{~nm}$ (defined media) in cuvettes of $1 \mathrm{~cm}$ light path in a Cecil CE292 spectrophotometer. Duplicate values greater than 0.1 were usually repeatable within $\pm 14 \%$. Duplicates less than 0.1 were repeatable within \pm 0.03 OD units.

Estimations. For measurements of enzyme activities, Pseudomonas $\mathrm{C12B}$ was grown in 1 litre Erlenmeyer flasks containing $400 \mathrm{ml}$ of medium. Bacteria were harvested in late-exponential phase by centrifuging $\left(10000 \boldsymbol{g}_{\mathrm{av}}\right.$, $15 \mathrm{~min}$ ) and resuspended in approx. $5 \mathrm{ml} 10 \mathrm{~mm} \cdot \mathrm{Tris} / \mathrm{HCl}$ buffer, $\mathrm{pH} \mathrm{7.5}$. Chilled cell suspensions were disrupted using a French pressure cell operating at $126 \mathrm{MPa}$ ( 3 passes per sample) and cell debris was removed by centrifugation $\left(10000 \mathrm{~g}_{\mathrm{av}}, 1 \mathrm{~h}\right)$. Protein content of the cell extracts was measured by the method of Bradford (1976), and alcohol dehydrogenase activities were assayed using $\mathrm{NAD}^{+}$as co-factor and one of a variety of primary and secondary alcohols as described previously (Barrett $e t a l ., 1980 \mathrm{~b}$ ). For duplicate growth experiments, enzyme assays were generally within $\pm 10 \%$ of the mean.

\section{RESULTS AND DISCUSSION}

\section{Growth experiments}

Pseudomonas $\mathrm{C} 12 \mathrm{~B}$ was able to grow on a wide variety of alcohols (Fig. 1). In a $24 \mathrm{~h}$ incubation period with primary alcohols (Fig. $1 a$ ), growth was most extensive with the $\mathrm{C}_{10}-\mathrm{C}_{12}$ group. No growth was apparent with nonan-1-ol within the $24 \mathrm{~h}$ growth period, but lower primary alcohols $\left(\mathrm{C}_{6}-\mathrm{C}_{8}\right)$ and the higher alcohol, tetradecan-1-ol, supported modest growth. As the carbon chain length was decreased below $C_{6}$, or increased above $C_{14}$, growth became progressively more feeble. Primary alcohols therefore fell into two groups: those of chain length $\mathrm{C}_{10}-\mathrm{C}_{12}$ supporting good growth, divided at $\mathrm{C}_{9}$ (no growth in $24 \mathrm{~h}$ ) from the shorter compounds giving moderate growth. A generally similar grouping was observed after $24 \mathrm{~h}$ growth on racemic secondary alkan-2-ols (Fig. 1b), with the exceptions that (i) the optimum chain-length was one carbon higher $\left(\mathrm{C}_{12}-\mathrm{C}_{13}\right)$ than for primary alcohols, (ii) the critical low-growth chain-length was also one carbon higher $\left(C_{10}\right)$ and (iii) all shorter alkan-2-ols gave very feeble growth. The reason why 

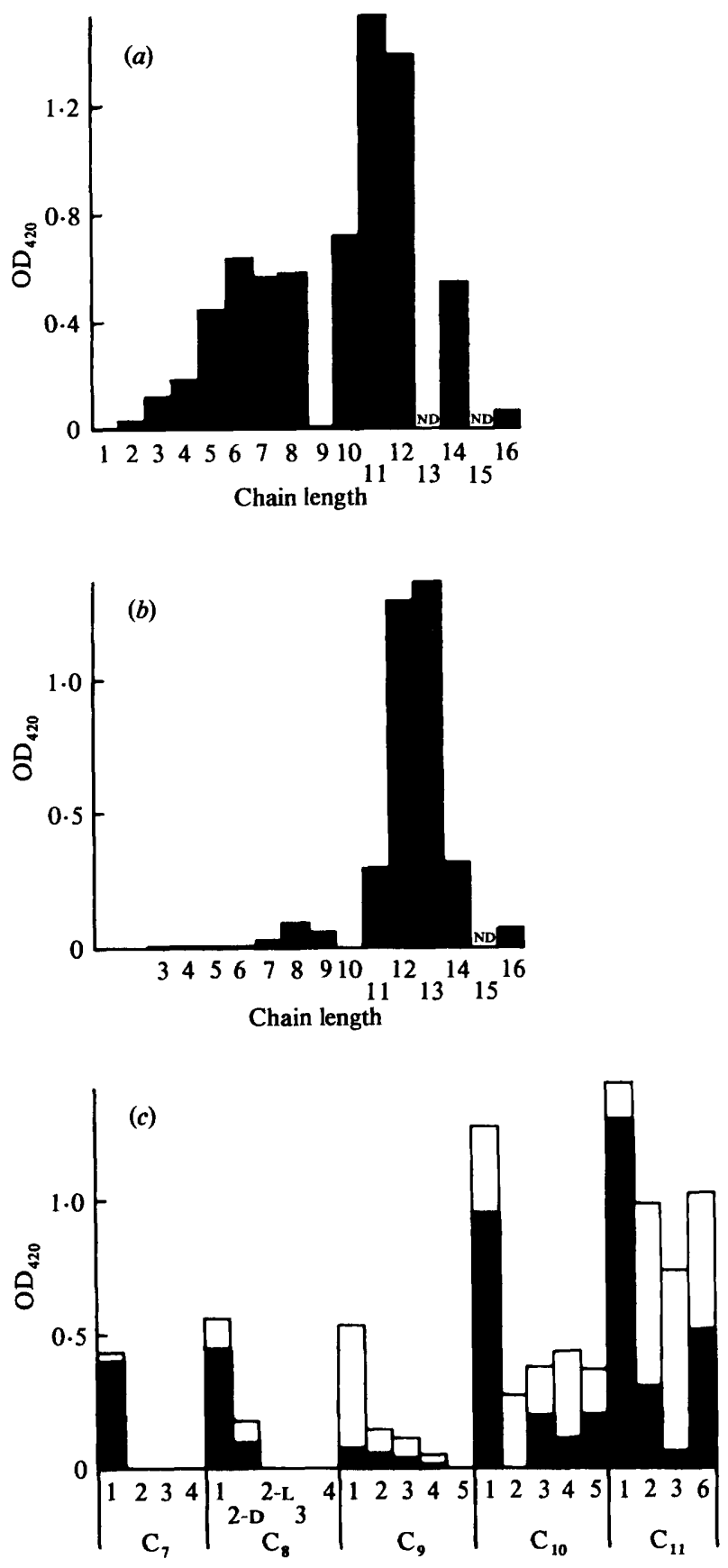

Fig. 1. Extent of growth of Pseudomonas $\mathrm{C12B}$ on various primary and secondary alcohols. Culture optical densities $(420 \mathrm{~nm})$ were measured after growth on $(a)$ homologous primary alcohols; $(b)$ homologous alkan-2-ols; $(c)$ isomeric heptanols $\left(C_{7}\right)$, octanols $\left(C_{8}\right)$, nonanols $\left(C_{9}\right)$, decanols $\left(C_{10}\right)$ and undecanols $\left(C_{11}\right)$. In $(c)$, the abscissa notation indicates the position of the $\mathrm{OH}$ group on the alkyl chain. Racemic mixtures of asymmetric secondary alcohols were used in all experiments except where indicated by the $D$ and $L$ notation. $\square$, Growth after $24 \mathrm{~h} ; \square$, further growth in the 24-48 h period. ND, Not determined. In the absence of added alcohol, culture optical densities remained below 0.01 . 
nonan-1-ol and decan-2-ol should yield such low growth rates compared with close homologues is not clear. However, the one carbon shift between patterns for primary and alkan-2-ols is very reminiscent of the shift in free energy of binding of primary and alkan-2-ols to alcohol dehydrogenases, and of the corresponding sulphate esters to alkylsulphatases (see Dodgson \& White, 1983, for a review). Such shifts have been interpreted as reflecting hydrophobic binding of only the longer alkyl segment attached to the hydroxylated or esterified carbon atom, so that, for example, binding of octan-2-ol $\left(\mathrm{C}_{6} \mathrm{H}_{13} \cdot \mathrm{CHOH} \cdot \mathrm{CH}_{3}\right)$ is equal to that of heptan-1-ol $\left(\mathrm{C}_{6} \mathrm{H}_{13} \cdot \mathrm{CHOH} \cdot \mathrm{H}\right)$, both binding via a $\mathrm{C}_{6}$ alkyl segment. The growth patterns for homologous primary and alkan-2-ols may be reflecting a similar hydrophobic contribution at one or more stages in alcohol uptake and/or metabolism.

Fig. $1 c$ shows the results of separate experiments in which growth after 24 and $48 \mathrm{~h}$ was measured for all primary and secondary isomers in the $\mathrm{C}_{7}-\mathrm{C}_{10}$ range, and also for some undecanols. Growth after $24 \mathrm{~h}$ on primary alcohols agreed with the pattern in Fig. $1 a$ with excellent growth on $\mathrm{C}_{10}$ and $\mathrm{C}_{11}$, modest growth on $\mathrm{C}_{7}$ and $\mathrm{C}_{8}$ and very little growth on $\mathrm{C}_{9}$ alcohols. Extending the incubation period to $48 \mathrm{~h}$ allowed further growth to occur; this was most noticeable for nonan-1-ol which yielded growth commensurate with its $\mathrm{C}_{8}$ and $\mathrm{C}_{7}$ homologues.

Similarly consistent results were obtained for alkan-2-ols, and again, in the extended $48 \mathrm{~h}$ growth period, decan-2-ol supported growth comparable with that observed for its lower homologues. Interestingly, in the case of octan-2-ol, Pseudomonas C12B grew on the D-isomer, albeit poorly, but not at all on the L-isomer. Unfortunately, resolved enantiomers of other secondary alcohols were not available. Growth also occurred with some higher secondary alcohols, and was most prolific with secondary undecanols (Fig. 1c).

While further studies are required to establish growth kinetic parameters (lag-period, doubling times, maximum growth) as a function of growth-supporting alcohol, two important points have emerged from these experiments: the versatility of Pseudomonas $\mathrm{C12B}$ in its capacity to grow on a wide range of alcohols, typical of those produced by the organism's alkylsulphatases when confronted with a mixture of alkyl sulphate surfactants, and the ability of Pseudomonas $\mathrm{C12B}$ to grow on certain symmetrical or near-symmetrical alcohols, implying the operation of an alcohol dehydrogenase that could act on the products of the S3 alkylsulphatase.

\section{Alcohol dehydrogenase activities}

Extracts of cells grown to late-exponential phase on a variety of carbon sources were assayed (using $\mathrm{NAD}^{+}$as co-factor) for ability to oxidize octan-1-ol, D- and L-octan-2-ol, DL-octan-3-ol, DL-octan-4-ol and the symmetrical nonan-5-ol. Growth on nutrient broth (Fig. 2a), pyruvate (Fig. $2 b$ ), or the primary alcohol undecan-1-ol (Fig. $2 c$ ) led to production of appreciable dehydrogenase activity towards octan-1-ol and D-octan-2-ol but not towards the other secondary alcohols tested. An almost identical pattern was evident for extracts of cells grown on other substrates based on a primary alkyl chain, namely undecanoate or SDS (not shown). The same activities were present following growth on racemic secondary alcohols (the 2-, 3- and 6undecanols, Fig. $2 d-f$ ) but a new feature now emerged: additional dehydrogenase activity was also detected towards the more symmetrical alcohols DL-octan-3-ol, DL-octan-4-ol and especially nonan-5-ol. Movement of the $\mathrm{OH}$ group in the growth-supporting alcohol towards the centre of the alkyl chain (undecanols substituted at C-2>C-3>C-6) had the effect of increasing the specific activity of extracts towards the symmetrical nonan-5-ol. Growth on undecan-6-ol and undecan-6-one produced closely similar patterns (Fig. $2 f, g$ ). It thus appeared that a new enzyme(s) with activity towards symmetrical alcohols was inducible by growth on secondary alcohols (and probably ketones), especially the more symmetrical isomers.

\section{Chain length specificities of alcohol dehydrogenase activities}

Pseudomonas $\mathrm{C12B}$ was grown to late-exponential phase in separate cultures using as carbon source hexan-1-ol, undecan-1-ol, undecan-6-ol or tridecan-2-ol. These alcohols were chosen as representatives of different types and for their ability to support good growth (Fig. 1). Cell extracts were prepared and assayed for dehydrogenase activity towards homologous series of primary alcohols, alkan-2-ols and symmetrical alcohols. 


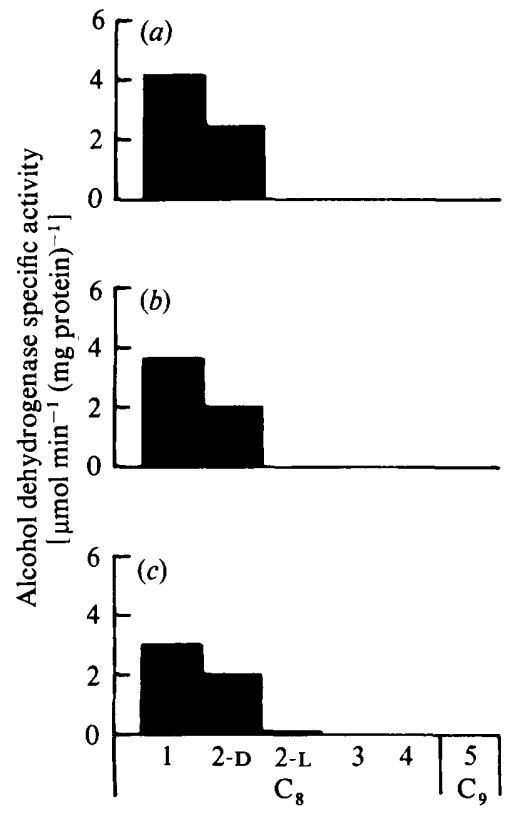

Assay substrate
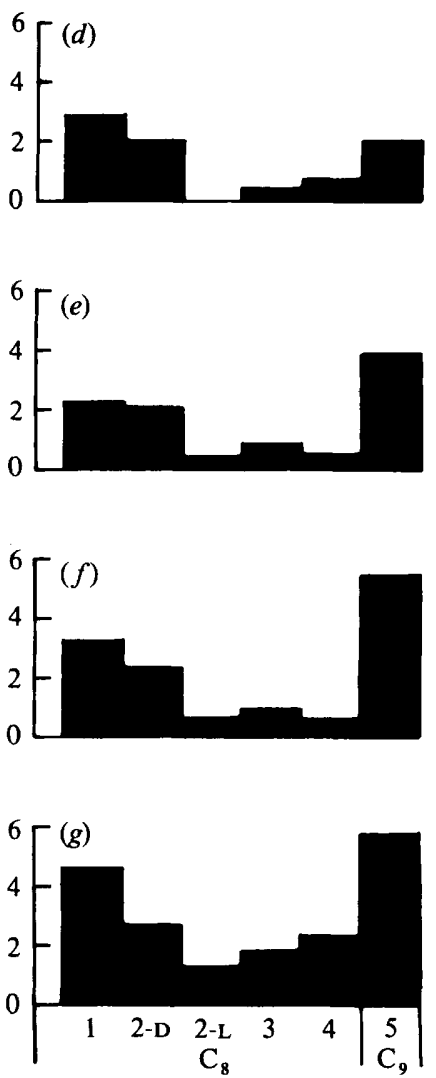

Assay substrate

Fig. 2. Primary and secondary alcohol dehydrogenase activities in extracts of Pseudomonas C12B after growth on (a) nutrient broth; $(b)$ pyruvate; $(c)$ undecan-1-ol; $(d)$ undecan-2-ol; $(e)$ undecan-3-ol; $(f)$ undecan-6-ol; $(g)$ undecan-6-one. Separate assays were done with $\mathrm{C}_{8}$ and $\mathrm{C}_{9}$ alcohols; the abscissa notation indicates the positional isomers used. Racemic mixtures of asymmetric secondary alcohols were used except were indicated by the $\mathrm{D}$ and $\mathrm{L}$ notation.

Extracts assayed for activity towards a range of primary alcohols $\left(\mathrm{C}_{2}-\mathrm{C}_{11}\right)$ all produced the same profile irrespective of growth-supporting alcohol, with two peaks of activity separated by a trough at $\mathrm{C}_{4}$ (Fig. $3 a-d$ ). The consistency of the enzyme activity-chain length profile with different alcohol growth-substrates was further evidence for constitutive synthesis of the primary alcohol dehydrogenase(s).

When the same extracts were assayed for activity towards alkan-2-ols, a quite different picture emerged (Fig. $3 e-h)$. Growth on primary alcohols $\left(\mathrm{C}_{6}\right.$ and $\mathrm{C}_{11}$, Fig. $\left.3 e, f\right)$ produced some activity towards $\mathrm{C}_{3}$ and $\mathrm{C}_{4}$ alkan-2-ols but this decreased as the chain-length was increased. Growth on secondary alcohols (undecan-6-ol or tridecan-2-ol, Fig. 3g, $h$ ), however, yielded good activities in the $\mathrm{C}_{3}-\mathrm{C}_{11}$ range, peaking in the $\mathrm{C}_{7}-\mathrm{C}_{8}$ region. The results clearly indicated induction of one or more secondary alcohol dehydrogenases when the organism was grown on secondary alcohols.

Dehydrogenase activity towards symmetrical alcohols was highest in extracts grown on the symmetrical ketone undecan-6-one (Fig. $2 g$ ). When fresh extracts of cells grown on undecan-6one were assayed for ability to oxidize homologous symmetrical alcohols, there was low activity towards heptan-4-ol, but more activity towards the $\mathrm{C}_{5}, \mathrm{C}_{9}$ and $\mathrm{C}_{11}$ homologues (Fig. $4 a$ ). There was also remarkably high activity towards propan-2-ol, possibly because this alcohol is symmetrical and an alkan-2-ol and therefore a substrate for both groups of secondary alcohol 

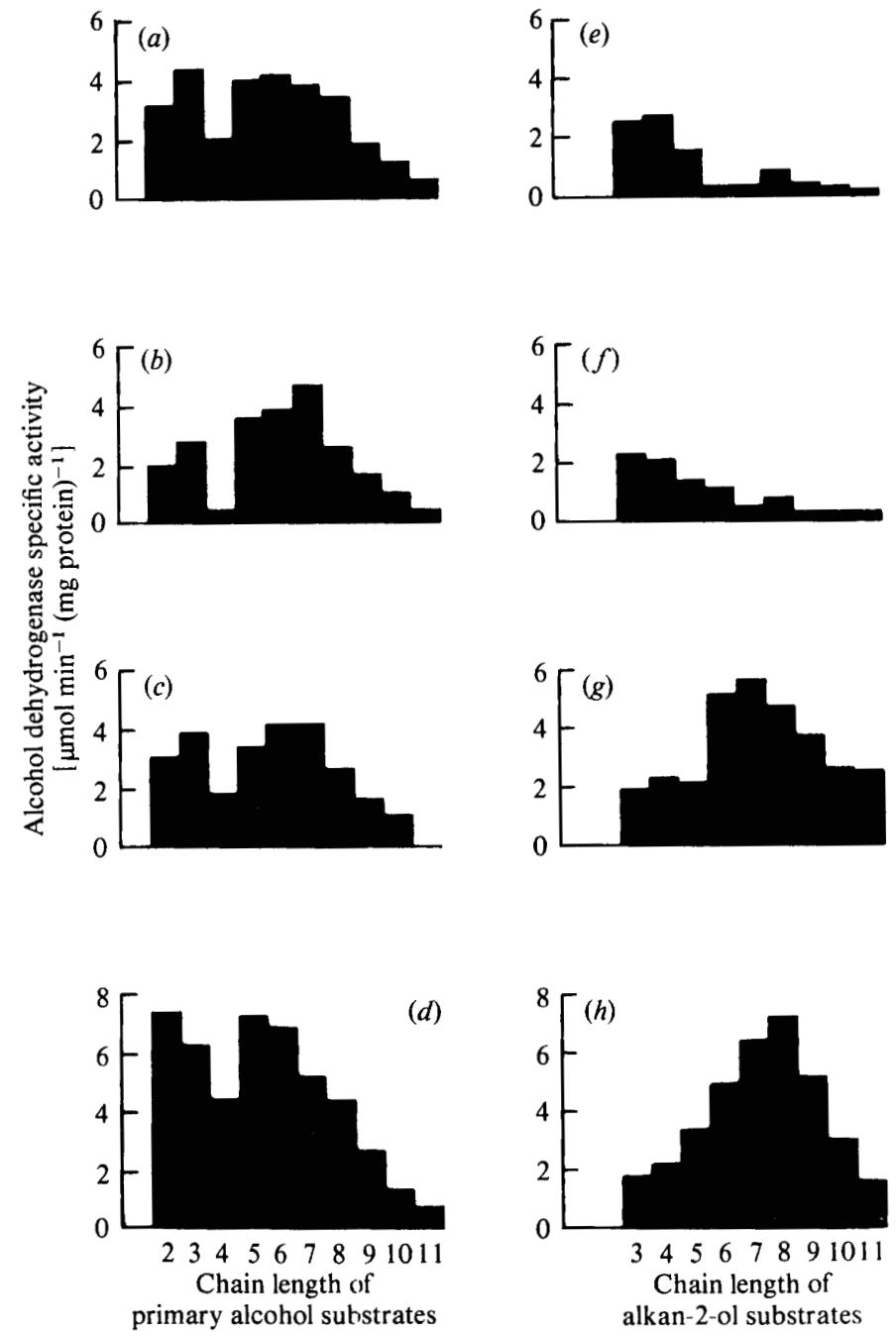

Fig. 3. Alcohol chain length specificity of primary alcohol dehydrogenase and alkan-2-ol dehydrogenase activities in extracts of Pseudomonas $\mathrm{C} 12 \mathrm{~B}$ grown on $(a, e)$ hexan-1-ol; $(b, f)$ undecan-l-ol; $(c, g)$ undecan-6-ol; $(d, h)$ tridecan-2-ol. $(a)-(d)$, Dehydrogenase activities towards homologous primary alcohols; $(e)-(h)$, dehydrogenase activities towards homologous alkan-2-ols.

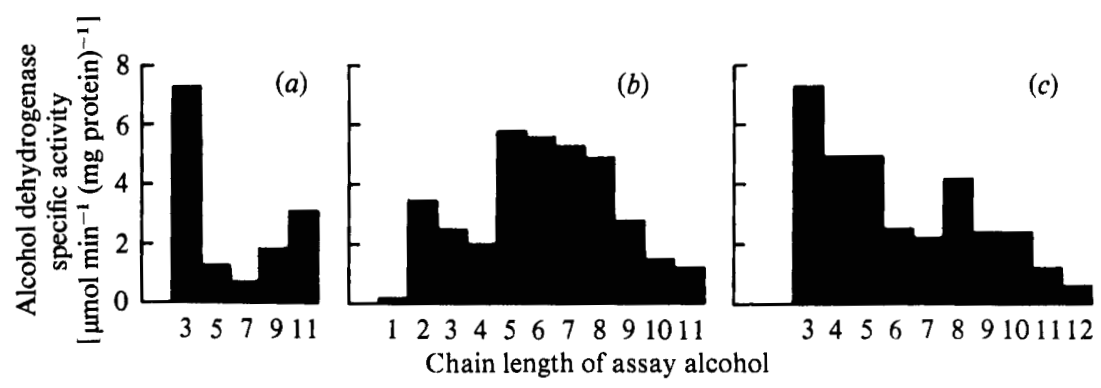

Fig. 4. Alcohol chain length specificity of (a) symmetrical, (b) primary and (c) alkan-2-ol alcohol dehydrogenase activities in extracts of Pseudomonas $\mathrm{C12B}$ grown on undecan-6-one. 
dehydrogenases (Fig. 4a,c). High activity towards propan-2-ol was accompanied by high activity towards its $\mathrm{C}_{4}$ and $\mathrm{C}_{5}$ homologues (Fig. $4 \mathrm{c}$ ), and in this respect, the profile differs from that of cells grown on undecan-6-ol (Fig. $3 g$ ) and tridecan-2-ol (Fig. $3 h$ ). At longer chain lengths $\left(>\mathrm{C}_{6}\right)$, however, a consistent pattern peaking around $\mathrm{C}_{7}-\mathrm{C}_{8}$ was observed for cells grown on undecan-6-one (Fig. 4c), undecan-6-ol (Fig. 3g) or tridecan-2-ol (Fig. 3h). Growth on undecan-6one also led to the usual pattern for primary alcohol dehydrogenase (Fig. $4 b$, cf. Fig. $3 a-d$ ).

Clearly (and not unexpectedly) Pseudomonas C12B produces a complex pattern of alcohol dehydrogenase activities, and several important features deserve comment. First, there was constitutive production of primary alcohol dehydrogenase activity that enabled the organism to oxidize alcohols liberated from primary alkyl sulphates by the P1 and P2 alkylsulphatases. Several other organisms are known to produce similar long-chain alcohol dehydrogenases (Dodgson \& White, 1983). Second, constitutive production of D-alkan-2-ol dehydrogenase enables the organism to oxidize the products of the S2 (L-specific) alkylsulphatase. The D-alkan2-ol dehydrogenase and the constitutive primary enzyme may be a single protein, as appeared to be the case in C. terrigena. Third, growth on secondary alcohols induces synthesis of an L-alkan2-ol dehydrogenase. Dehydrogenase specificity for simple L-alkan-2-ols was first discovered in C. terrigena (Barrett et al., 1980b) and has since been found by Hou et al. $(1981 a, b)$. In Pseudomonas $\mathrm{C12B}$, the activity provides a route for oxidation of the bond-inverted products of the S1 (D-specific) alkylsulphatase. These sulphatase-dehydrogenase pairings have parallels in C. terrigena (see Introduction). Fourth, an enzyme with high activity towards symmetrical alcohols was inducible by growth on secondary (especially symmetrical) alcohols. The appearance of the symmetrical alcohol dehydrogenase always coincided with induction of the Lalcohol dehydrogenase and these may in fact be activities of a single broad-specificity enzyme. The corresponding activities in $C$. terrigena also appeared to be coincident, although the symmetrical activity was relatively weak. Symmetrical alcohol dehydrogenase activity has been reported in another Pseudomonas sp., but this lacked activity towards alkan-2-ols (Niehaus et al., 1978). Discovery of symmetrical alcohol dehydrogenase activity in Pseudomonas C12B should be viewed in the light of earlier work from these laboratories on the inducible S3 alkylsulphatase that acts on symmetrical alkyl sulphates to yield the corresponding secondary alcohols. Induction of the $\mathrm{S} 3$ enzyme is intriguing because it requires a combination of a long-chain secondary alkyl sulphate and a secondary alcohol (Dodgson et al., 1974). The observation that growth on secondary alcohols served also to induce the dehydrogenase required to oxidize the symmetrical alcohol products of S3 alkylsulphatase action suggested that synthesis of the enzymes might be co-ordinated. Work to explore the genetic relationships among the various alkylsulphatases and alcohol dehydrogenases has been initiated.

Finally, the observation that extracts of organisms grown on DL-undecan-2-ol (Fig. $2 d$ ) had dehydrogenase activity towards D- but not L-octan-2-ol was consistent with the capacity of the organism to grow on D-octan-2-ol but not on the corresponding L-isomer (Fig. 1c). Moreover these observations collectively suggested that growth on racemic DL-alkan-2-ols should result in assimilation of the D-isomer only, and simultaneous accumulation of the L-isomer in the culture medium. The system would thus afford a convenient method of resolving L-isomers of racemic alkan-2-ols (an otherwise difficult chemical resolution) and this prospect is currently being pursued.

This work was supported by a SERC Research Project Grant to K. S. D. which is gratefully acknowledged.

\section{REFERENCES}

Barrett, C. H., Dodgson, K. S. \& White, G. F. $(1980 a)$. Further studies on substrate and inhibitor specificity of the stereospecific CS2 secondary alkylsulphohydrolase of the detergent-degrading micro-organism Comamonas terrigena. Biochemical Journal 191, 467-473.

Barrett, C. H., Dodgson, K. S., White, G. F. \& PAYNe, W. J. (1980 $b$ ). Preliminary observations on alcohol dehydrogenases in Comamonas terrigena that exhibit stereospecificity towards secondary alcohols. Biochemical Journal 187, 703-709.

Barrett, C. H., Dodgson, K. S. \& White, G. F. (1981). Specificity and other properties of an alcohol dehydrogenase purified from Comamonas terrigena. Biochimica et biophysica acta 661, 74-86.

BRADFORD, M. M. (1976). A rapid and sensitive 
method for the quantitation of microgram quantities of proteins utilizing the principle of protein-dye binding. Analytical Biochemistry 72, 248-254.

Dodgson, K. S. \& White, G. F. (1983). Some enzymes involved in the microbial degradation of sulphated surfactants. In Topics in Enzyme and Fermentation Biotechnology, vol. 7, pp, 90-155. Edited by A. Wiseman. Chichester: Ellis Horwood.

Dodgson, K. S., Fitzgerald, J. W. \& Payne, W. J. (1974). Chemically defined inducers of alkylsulphatases present in Pseudomonas C12B. Biochemical Journal 138, 53-62.

Fitzgerald, J. W. \& Payne, W. J. (1972). Induction in a Pseudomonas species of sulphatases active on short chain alkylsulphates. Microbios 5, 87-100.

hou, C. T., Patel, R., Laskin, A. I., Barnabe, N. \& MARCZAK, I. (1981a). Substrate specificity and stereospecificity of nicotinamide adenine dinucleotide-linked alcohol dehydrogenases from methanolgrown yeasts. Applied and Environmental Microbiology 41, 829-832.

hou, C. T., Patel, R., Barnabe, N. \& Marczak, I. $(1981 b)$. Stereospecificity and other properties of a novel secondary-alcohol-specific alcohol dehydrogenase. European Journal of Biochemistry 119, 359364.

Hugh, R. (1965). A comparison of Pseudomonas testosteroni and Comamonas terrigena. International Bulletin of Bacteriological Nomenclature and Taxonomy 15, 125-132.
Lijmbach, G. W. M. \& Brinkhuis, E. (1973). Microbial degradation of secondary $n$-alkyl sulfates and secondary alcohols. Antonie van Leeuwenhoek 39, 415-423.

Matcham, G. W. J., Dodgson, K. S. \& Fitzgerald, J. W. (1977). Purification, properties and cellular localization of the stereospecific CS2 secondary alkylsulphohydrolase of Comamonas terrigena. Biochemical Journal 167, 723-729.

Niehaus, W. G., Frielle, T. \& Kingsley, E. A. (1978). Purification and characterization of a secondary alcohol dehydrogenase from a pseudomonad. Journal of Bacteriology 134, 177-183.

Palleroni, N. J., Kunisawa, R., Contopoulou, R. \& DoudorofF, M. (1973). Nucleic acid homologies in the genus Pseudomonas. International Journal of Systematic Bacteriology 23, 333-339.

PAYNE, W. J. (1963). Pure culture studies of the degradation of detergent compounds. Biotechnology and Bioengineering 5, 355-365.

Payne, W. J. \& Feisal, V. E. (1963). Bacterial utilization of dodecyl sulphate and dodecyl benzene sulfonate. Applied Microbiology 11, 339-344.

Stanier, R. Y., Palleroni, N. J. \& Doudoroff, M. (1966). The aerobic pseudomonads: a taxonomic study. Journal of General Microbiology 43, 159-271.

Williams, J. P., Mayberry, W. R. \& Payne, W. J. (1966). Metabolism of linear alcohols with various chain lengths by a Pseudomonas species. Applied Microbiology 14, 156-160. 